DOI: 10.12957/demetra.2016.16779

\title{
Oferta de sódio oriundo de alimentos industrializados em restaurantes institucionais
}

\section{Sodium amounts in industrialized food in institutional restaurants}

\author{
Adriana Maria de Oliveira Brito \\ Mônica Glória Neumann Spinelli' \\ 1 Universidade Presbiteriana Mackenzie, Centro \\ de Ciências Biológicas e da Saúde, Curso de \\ Nutrição. São Paulo-SP, Brasil. \\ Correspondência / Correspondence \\ Adriana Maria de Oliveira Brito \\ E-mail: adriana.mob@gmail.com
}

\section{Resumo}

Em benefício da saúde dos indivíduos, o consumo de sódio per capita deve ser restringido. Para isso é importante, também, que os clientes de unidades de alimentação e nutrição (UAN) conheçam o que de fato têm em seus pratos, para que possam fazer suas opções. Objetivo: A pesquisa pretendeu descrever a oferta de sódio oriunda de alimentos industrializados em restaurantes institucionais no estado de São Paulo, tendo em perspectiva a sugestão da Organização Mundial da Saúde (OMS), de consumo diário inferior a $5 \mathrm{~g}$ de cloreto de sódio, ou seja, $2 \mathrm{~g}$ de sódio. Método: A partir dos documentos de controle de estoque de cada UAN, foram coletadas informações a respeito de produtos industrializados utilizados no período de 30 dias. A quantidade de sódio apurada foi resultante da multiplicação da informação contida nos rótulos dos alimentos industrializados por sua quantidade utilizada para a elaboração das preparações oferecidas em almoços. A partir dessa informação e do número de refeições servidas, foi apurada a quantidade média de sódio oferecida por esses produtos a cada cliente, por dia. A média foi comparada à sugestão da OMS. Resultados: O teor de sódio contido nos alimentos industrializados utilizados nas preparações fornecidas pelas UANs, na média, é muito maior do que o estimado para uma refeição-almoço, atingindo até o nível 2,5 vezes o valor recomendado pela OMS. Conclusão: Dado que todas as unidades avaliadas apresentaram resultados semelhantes, fazse necessária a revisão das técnicas de preparo utilizadas na UAN, com revisão dos ingredientes usados nas preparações.

Palavras-chave: Alimentação Coletiva. Alimentos Industrializados. Almoço. Sal. Sódio. Recomendações Nutricionais. 


\section{Abstract}

In benefit of the health of individuals, the consumption of sodium per capita should be restrained, so it is also important that the clients at foodservice units (UAN) know what is in fact on their plates, so that they can make their choices. Objective: This research aimed to point out the impact of industrialized products as to the amounts of sodium offered to users of these units in the state of São Paulo, considering the suggestion of the World Health Organization, of a daily consumption inferior to $5 \mathrm{~g}$ of sodium chloride a day, or $2 \mathrm{~g}$ of sodium. Método: Information concerning industrialized products utilized in a period of thirty days have been collected from the stock control documents from each unit. The calculated quantity of sodium was a result of the multiplication of the information in the labels of the industrialized food, by the amount used to prepare plates served for lunch. Given that information and the number of served meals, the average amount of sodium offered daily by those products per client was calculated. This average was compared to the WHO's suggestion. Results: The content of sodium in industrialized food used in the preparations supplied by the nutrition units is, in average, greater than the previously valued amount for a lunch meal, reaching up to 2.5 times the pre-stablished suggestion. Conclusion: One must reduce the content of this mineral, raising the awareness of the population, of food business managers and of the Brazilian government.

Key words: Collective Feeding. Industrialized Food. Lunch. Salt. Sodium. Recommended Dietary Allowances.

\section{Introdução}

A alimentação saudável é importante porque contribui para a proteção contra as doenças crônicas não transmissíveis (DCNTs) que são potencialmente fatais, como hipertensão, diabetes, acidente vascular cerebral, doenças cardíacas e alguns tipos de câncer. Essas doenças estão entre as principais causas de incapacidade e morte no Brasil e em outros países. ${ }^{1}$

As DNCTs derivadas da elevação da pressão arterial, ${ }^{2}$ osteoporose,${ }^{3}$ câncer gástrico, ${ }^{4}$ síndrome metabólica, ${ }^{5}$ etc são problemas que podem ter como origem uma alimentação inadequada, na qual há ingestão excessiva de sal, um ingrediente muito importante na culinária mundial, composto pelos elementos químicos sódio (na proporção de 40\%) e cloro (na proporção de 60\%). ${ }^{6}$ É o sódio que, quando ingerido em excesso, pode causar problemas à saúde do indivíduo. Todavia, se consumido em quantidades adequadas, esse mineral continuará a exercer apenas as atividades importantes para o organismo humano, quais sejam: controle da pressão arterial, auxílio na regulação do 
volume plasmático, na condução dos impulsos nervosos e na contração muscular. ${ }^{7}$ Dessa forma, reduzir a ingestão de sódio é uma medida válida para diminuir os índices de hipertensão arterial (HA) e, por consequência, diminuir a morbimortalidade causada por doenças crônicas.

A Organização Mundial da Saúde (OMS) recomenda o consumo diário inferior a $5 \mathrm{~g}$ de cloreto de sódio por dia, ou seja, $2 \mathrm{~g}$ de sódio ${ }^{8}$ no entanto, estudos indicam que o consumo geral é muito maior. A Pesquisa de Orçamento Familiar 2008/2009 mostra que parte da população brasileira consome 3,6g de sódio diariamente. ${ }^{9}$ Em Portugal, estudos indicam consumo variando entre 9 a 12 gramas de sal por dia, ${ }^{10}$ assim como nos Estados Unidos. ${ }^{11}$ No Reino Unido, a média de consumo diário é de $8, \lg ^{12}$

Assim, para que seja possível encontrar alternativas para a diminuição do consumo desse nutriente, faz-se necessário conhecer suas fontes, que podem ser diversas, tais como: o sal de adição, o sal e/ou sódio contido em produtos industrializados, o glutamato monossódico, o bicarbonato de sódio, o edulcorante, o aditivo, etc., além do sódio contido naturalmente nos alimentos. ${ }^{13}$

É de conhecimento comum que a quantidade desse nutriente ingerido por conta de alimentos industrializados é muito grande. Sichieri et al. apuraram, em sua pesquisa, que os alimentos industrializados contêm até 20 vezes mais sal do que os alimentos naturais, implicando acréscimo considerável de sódio na refeição. ${ }^{14}$ Preocupado com esses fatos, o Ministério da Saúde elaborou o Plano de Redução do Sódio em Alimentos Processados, um componente do Plano de Redução do Consumo de Sal pela População Brasileira, baseado em experiências internacionais e em execução desde abril de 2011. O plano prevê a redução do consumo para $5 \mathrm{~g}$ de sal per capita por dia - 2.000 mg de sódio - até 2020 e deve ser atingido por redução voluntária, por parte dos produtores, do teor de sódio nos alimentos processados. ${ }^{15}$

No entanto, além das ações voltadas para a produção de alimentos processados, outras podem ser estabelecidas em benefício da saúde dos indivíduos. Assim, considerando um aumento no consumo da alimentação fora do domicílio na área urbana do Brasil, ${ }^{9}$ como naqueles restaurantes inseridos em hospitais, indústria, empresas, escolas, entre outros, e designados de unidades de alimentação e nutrição ${ }^{16}(\mathrm{UAN})$, torna-se relevante pensar em ações dirigidas para esses estabelecimentos, como, por exemplo, diminuição da quantidade de alimentos processados nas preparações e escolha de gêneros alimentícios que contenham menor quantidade de sódio. Outra medida seria nestes estabelecimentos informar aos comensais os ingredientes que compõem a preparação, com destaque para aqueles com maior teor de sódio, em acordo com os padrões indicados pela OMS.

A redução do consumo de sódio na população mediante estratégias de saúde pública rentáveis é, assim, considerada relevante para a manutenção da saúde e prevenção das DCNTs. Em virtude do que foi mencionado, esta pesquisa pretende descrever a oferta de sódio oriunda de alimentos industrializados em UAN do estado de São Paulo, para que seja reconhecido o papel colaborativo na redução do consumo de sódio que pode acontecer através de ações por parte dessas entidades. 


\section{Método}

Trata-se de estudo observacional transversal, com amostra de conveniência, em UANs nas cidades de São Paulo, São Bernardo do Campo e Osasco. Foram convidados seis restaurantes institucionais para participar da pesquisa, escolhidos pela facilidade de acesso aos responsáveis pelas empresas contratantes do serviço de alimentação, sendo que quatro deles aceitaram a proposta.

Dos documentos de controle de estoque de cada restaurante, foram coletadas informações sobre os produtos industrializados e respectivas quantidades utilizadas, sendo a coleta de dados realizada pela pesquisadora, no período compreendido entre abril a julho de 2014. Assim, a quantidade de sódio foi apurada, para cada alimento processado utilizado na unidade, a partir das informações nutricionais dos rótulos e da quantidade de cada produto informado pela UAN.

Calculado o valor de sódio dos alimentos industrializados utilizados no dia, dividiu-se essa quantidade total pelo número de refeições produzidas no dia, para obtenção da quantidade média per capita ofertada por cada item do cardápio. A somatória desses dados forneceu a quantidade média per capita de sódio na refeição ofertada pelos produtos industrializados. Esse procedimento foi realizado por 30 dias corridos em cada unidade.

Tomaram-se como parâmetros para comparação a recomendação da OMS de ingestão menor do que $5 \mathrm{~g}$ por dia de sal e a distribuição do valor energético total (VET) diário, que considera que o almoço corresponde a cerca de 35\% do VET (o Programa de Alimentação do Trabalhador estima uma variação entre 720 e $960 \mathrm{mg}$ de sódio para o almoço). ${ }^{16}$ Assim, considerando o proposto pela OMS, tem-se que o limite por dia esperado de consumo total de sódio seja igual a $2.000 \mathrm{mg}$ (o sódio contido em $5 \mathrm{~g}$ de sal). Se um almoço representa 35\% do VET, então o consumo máximo desejado para essa refeição é de $700 \mathrm{mg}$.

$\mathrm{Na}$ exposição dos dados, foram listados os ingredientes que mais contribuíram para o fornecimento de $\mathrm{Na}$, em ordem decrescente de oferta, até que $75 \%$ da quantidade total de sódio do mês, ofertado por cada unidade, estivesse representada de modo a se conhecer os alimentos fontes de sódio mais utilizados dentro de cada UAN.

O presente trabalho foi realizado de acordo com a Resolução CNS/MS nº 466/2012, sendo que as instituições em que os dados foram coletados, após esclarecimento sobre os objetivos da pesquisa, assinaram o Termo de Consentimento Livre e Esclarecido.

\section{Resultado e Discussão}

O cômputo do teor de sódio contido nos alimentos industrializados utilizados nas preparações fornecidas pelas UANs visitadas revelou que, na média, a oferta desse mineral, por meio dessa 
fonte, é maior do que o recomendado para uma refeição-almoço, considerando a sugestão feita pela OMS, de ingestão menor do que $5 \mathrm{~g}$ por dia de sal e distribuição do valor energético total diário, que considera que o almoço corresponde a cerca de $35 \%$ do VET. ${ }^{8}$ Em valores médios, foi apurado que a unidade "1" ofereceu 1.583,69 mg de sódio em um almoço; a unidade "2" ofertou 970,68 mg; a unidade "3", 1.075,05 mg; e a unidade "4", 1.769,21 mg. Segundo a ANVISA:17

[...] as metas de cada estabelecimento devem partir da elaboração das fichas técnicas preparatórias para análise do teor de sal e sódio e a redução gradual dos mesmos até a oferta média nas grandes refeições de 920 mg para o somatório das porções das preparações servidas.

O trabalho de Borjes, Tasca \& Zamprogna ${ }^{18}$ apurou a oferta de sódio proveniente dos produtos industrializados igual a 910mg, valor inferior aos encontrados neste trabalho.

É de se salientar que o consumo de Na não se dá somente através dessa fonte e, também por isso, é de extrema importância que se avalie a necessidade de uso de ingredientes com elevado teor deste mineral na elaboração de qualquer cardápio, além de se considerar a relação custobenefício da utilização desses itens.

Há UANs que trabalham com programas que pretendem salvaguardar a saúde dos comensais e, por isso, desenvolvem campanhas que vão desde prestar informação sobre determinado alimento ou nutriente até implementar novas opções no cardápio para incentivar ou desestimular seu consumo. Das unidades participantes deste estudo, a "2" possui esse tipo de ação e é a que apresentou a menor média de oferta de Na no período avaliado. Nessa unidade, há um programa que visa promover a alimentação saudável do trabalhador, e dentro dele há um projeto que pretende diminuir o consumo de sódio por parte de seus colaboradores. Ele é executado frequentemente com a eliminação de saleiros nas mesas, projetos de conscientização dos consumidores da UAN com a distribuição de panfletos informativos e palestras ministradas por nutricionistas. A partir da apresentação do resultado desta pesquisa, adotaram também o corte de fornecimento de linguiça e gelatina diet, por exemplo.

Conforme pode ser observado na figura 2, essa mesma unidade manteve o nível de oferta próximo aos $700 \mathrm{mg}$, com alguns dias abaixo dos 700mg e com outros bastante elevados. Tal fato ocorreu em função da preparação do dia. Por exemplo, no almoço de número 10 foi oferecida linguiça de frango, o que acabou gerando a oferta de 993,24 mg de Na por porção, já que foi ofertado um total de $60 \mathrm{~kg}$ desse produto a 621 comensais.Também nesse dia foi utilizado caldo de carne industrializado, o que somou 488,49 mg de sódio por porção à preparação à qual foi acrescentado, tendo em vista que foi utilizado, no total, um quilo desse produto para o mesmo número de clientes. 


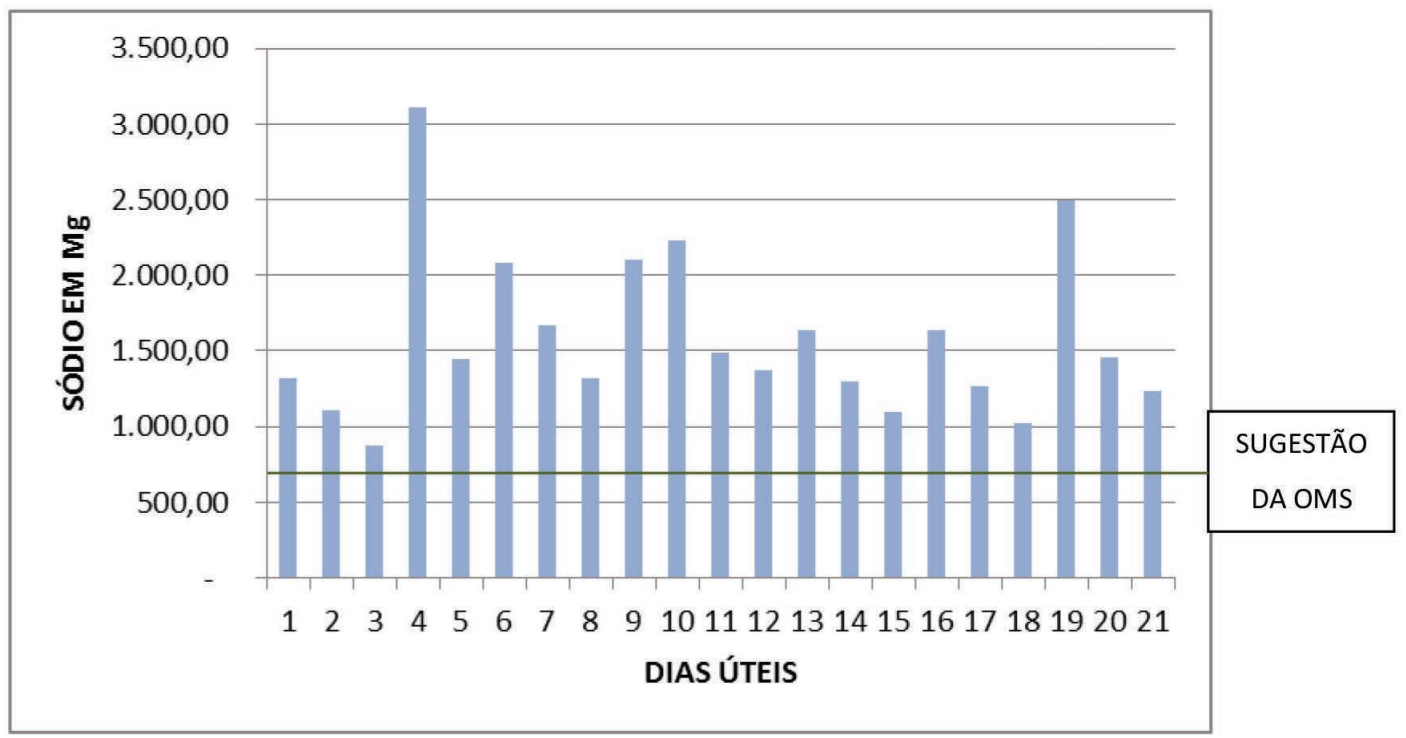

Figura 1. Oferta diária de sódio (em mg) nos dias úteis compreendidos entre 02/05/14 a 30/05/14 da unidade "1", comparada à sugestão da OMS.

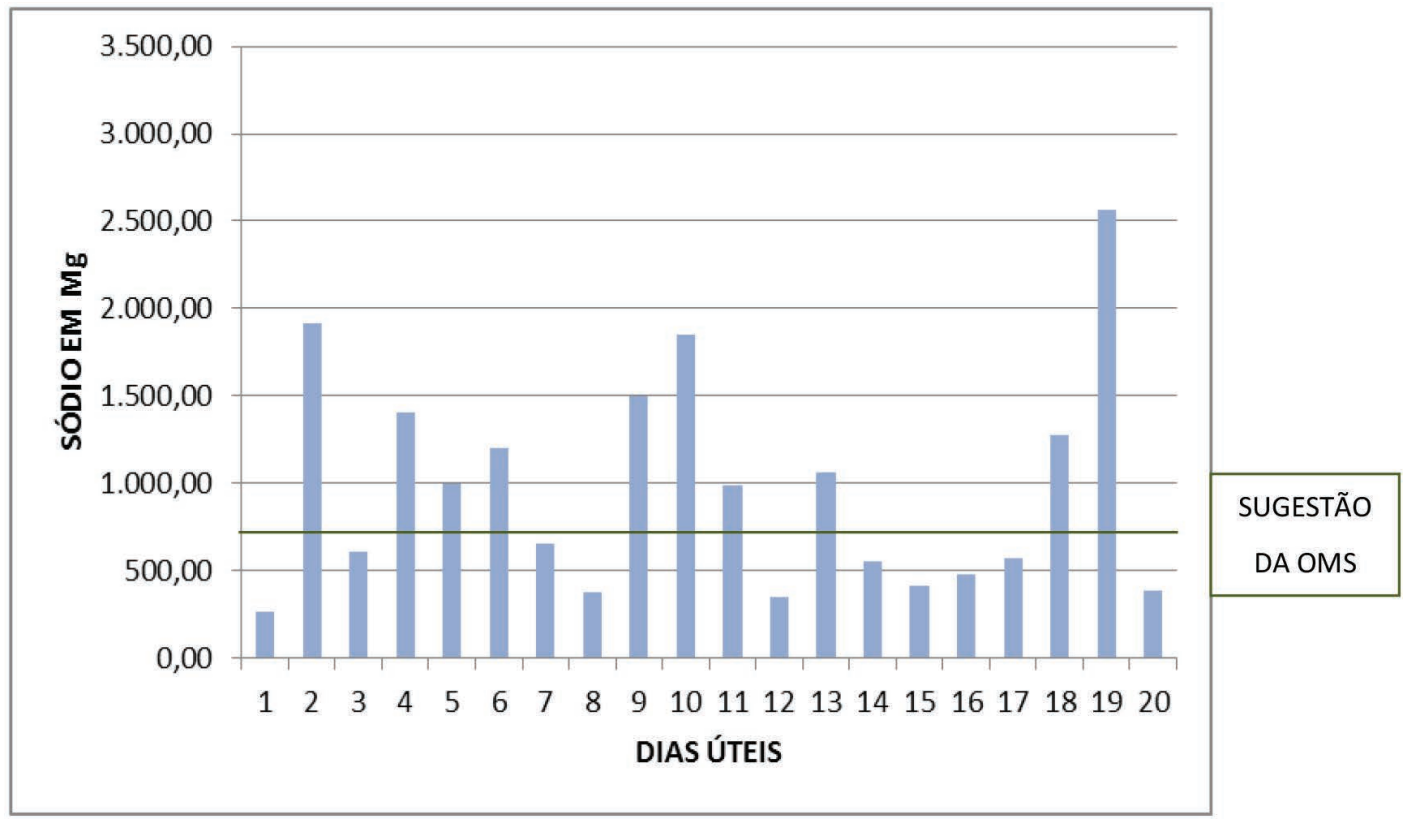

Figura 2. Oferta diária de sódio (em mg) nos dias úteis compreendidos entre 01/04/14 a 30/04/14 da unidade “2”, comparada à sugestão da OMS. 


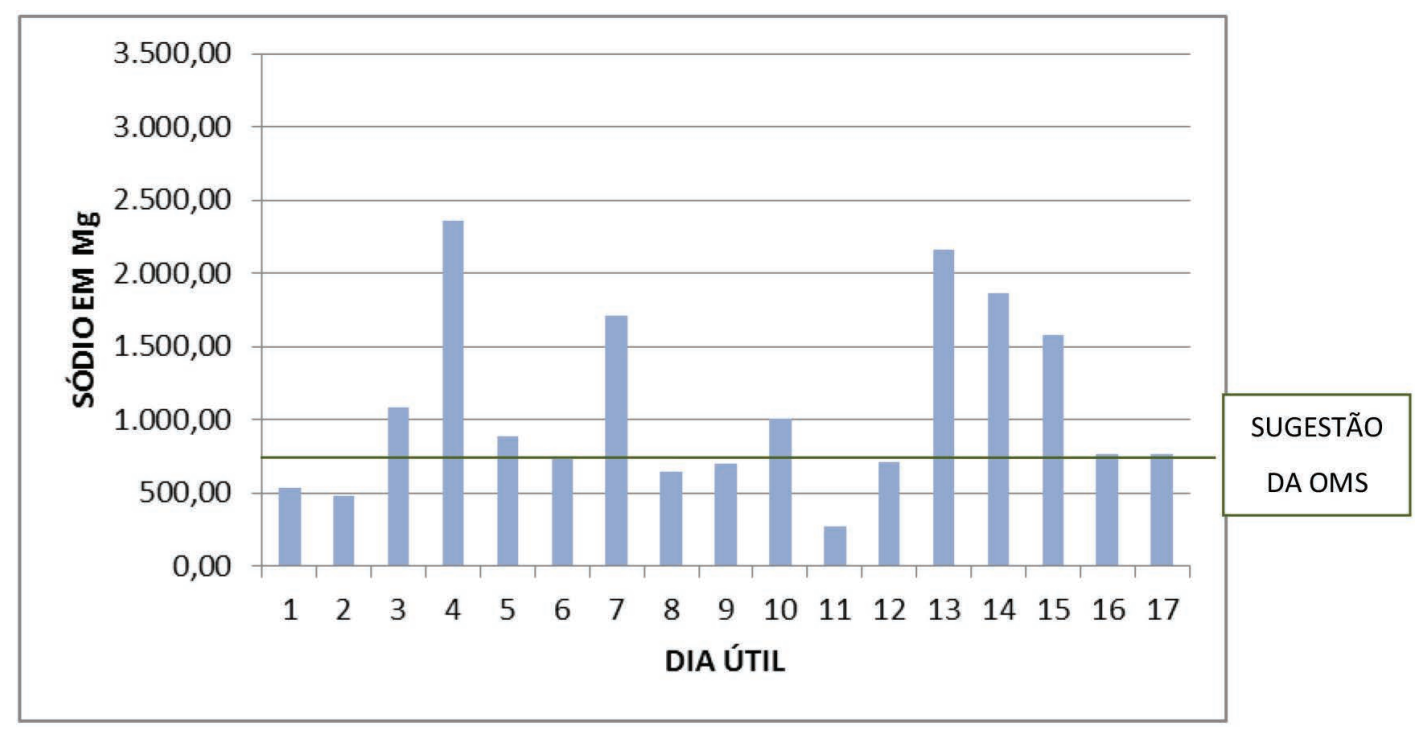

Figura 3. Oferta diária de sódio (em mg) nos dias úteis compreendidos entre 04/06/14 a 30/06/14 da unidade “3”, comparada à sugestão da OMS.

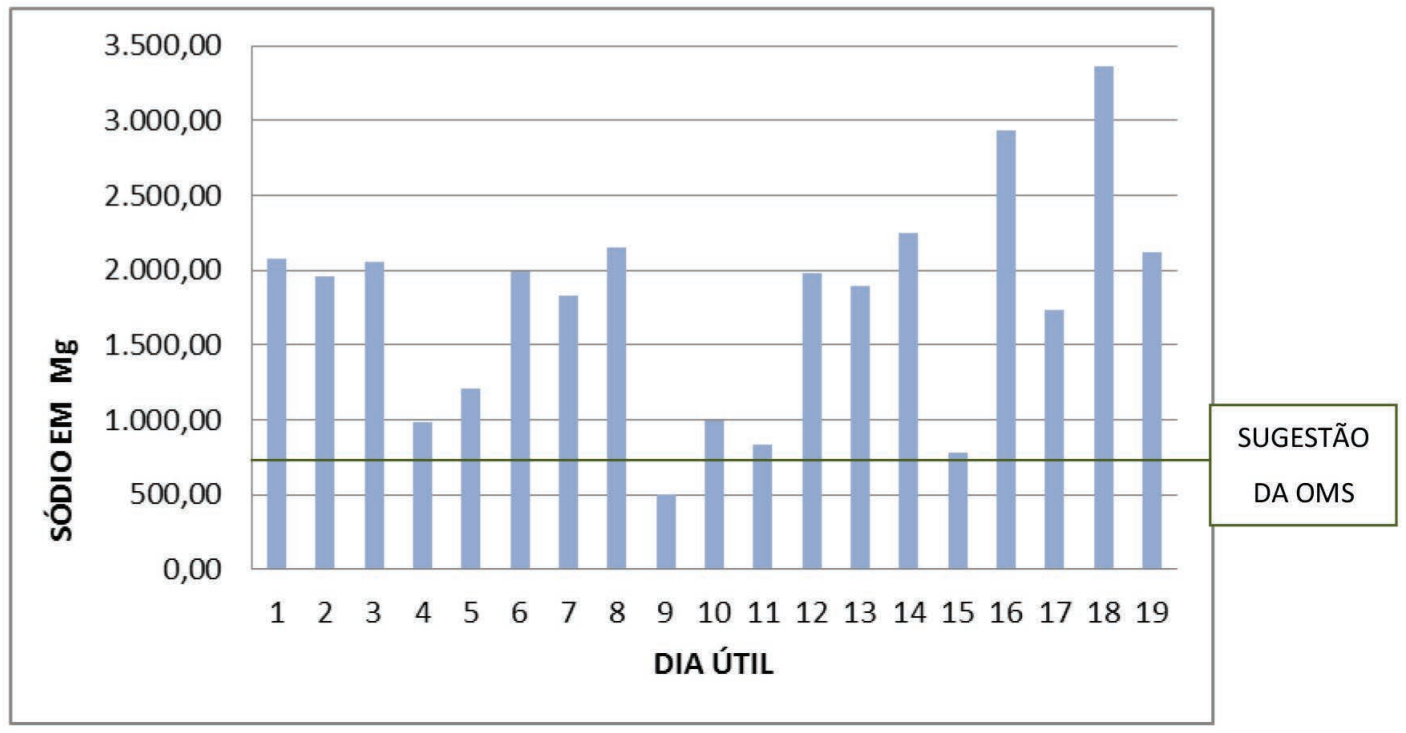

Figura 4. Oferta diária de sódio (em mg) nos dias úteis compreendidos entre 13/06/14 a 14/07/14 da unidade “4”, comparada à sugestão da OMS. 
Essa variação ocorre em todas as unidades, como se vê no gráfico 1, e por razões semelhantes.

As tabelas 1 a 4 mostram os produtos industrializados que mais contribuíram para a oferta de sódio. São mostrados os itens que, somados, chegam a aproximadamente $75 \%$ do teor de $\mathrm{Na}$ oferecido no mês avaliado.

Tabela 1. Alimentos industrializados que mais contribuíram para a quantidade de $\mathrm{Na}$ ofertada nos almoços da Unidade “1”, situada na cidade de São Paulo-SP, no período de 02 a 30/05/2014.

\begin{tabular}{l|c|c|c|c}
\hline \multicolumn{1}{c|}{ ALIMENTO } & $\begin{array}{c}\text { Na Per } \\
\text { Capita por } \\
\text { mês (mg) }\end{array}$ & $\begin{array}{c}\text { Número } \\
\text { de dias } \\
\text { de oferta }\end{array}$ & $\begin{array}{c}\text { Na per capita } \\
\text { por dia (mg) }\end{array}$ & $\begin{array}{c}\text { Oferta de Na } \\
\text { comparada à } \\
\text { sugestão da } \\
\text { OMS (\%) }\end{array}$ \\
\hline QUEIJO PARMESÃO & 5.408 & 21 & 258 & $43 \%$ \\
MOLHO ROSÊ & 3.879 & 17 & 228 & $38 \%$ \\
MOLHO ITALIANO & 3.115 & 17 & 183 & $31 \%$ \\
LINGUIÇA DE FRANGO & 2.215 & 6 & 369 & $62 \%$ \\
MOLHO PARMESÃO & 1.712 & 21 & 82 & $14 \%$ \\
MARGARINA VEGETAL & 1.640 & 20 & 82 & $14 \%$ \\
GELATINA FRUTA & 1.479 & 19 & 78 & $13 \%$ \\
MAIONESE & 1.124 & 4 & 281 & $47 \%$ \\
CARNE SECA & 932 & 4 & 233 & $39 \%$ \\
SALSICHA DE FRANGO & 834 & 6 & 139 & $23 \%$ \\
AZEITONA PRETA & 801 & 13 & 62 & $10 \%$ \\
EXTRATO TOMATE & 661 & 13 & 51 & $8 \%$ \\
QUEIJO MUÇARELA & 592 & 1 & 592 & $99 \%$ \\
NHOQUE BATATA PRÉ-COZ. & 591 & 21 & 28 & $5 \%$ \\
\hline
\end{tabular}


Tabela 2. Alimentos industrializados que mais contribuíram para a quantidade de Na ofertada nos almoços da Unidade "2", situada na cidade de São Paulo-SP, no período de 01 a $30 / 04 / 2014$.

\begin{tabular}{l|c|c|c|c}
\hline \multicolumn{1}{c|}{ ALIMENTO } & $\begin{array}{c}\text { Na per } \\
\text { capita por } \\
\text { mês (mg) }\end{array}$ & $\begin{array}{c}\text { Número } \\
\text { de dias } \\
\text { de oferta }\end{array}$ & $\begin{array}{c}\text { Na per } \\
\text { capita por } \\
\text { dia (mg) }\end{array}$ & $\begin{array}{c}\text { Oferta de Na } \\
\text { comparada à } \\
\text { sugestão da } \\
\text { OMS (\%) }\end{array}$ \\
\hline CALDO DE LEGUMES & $2.646,35$ & 5 & 529,27 & $88 \%$ \\
AMACIANTE DE CARNE & $2.156,43$ & 2 & $1.078,22$ & $180 \%$ \\
MOLHO SOJA & $1.946,23$ & 13 & 149,71 & $25 \%$ \\
QUEIJO PARMESÃO & $1.460,51$ & 17 & 85,91 & $14 \%$ \\
PÃO FRANCÊS & $1.267,29$ & 7 & 181,04 & $30 \%$ \\
MARGARINA VEGETAL C/ SAL & $1.211,18$ & 5 & 242,24 & $40 \%$ \\
LINGUIÇA DE FRANGO & 993,24 & 1 & 993,24 & $166 \%$ \\
CALDO DE CARNE & 646,13 & 1 & 646,13 & $108 \%$ \\
AZEITONA PRETA S/ CAROÇO & 603,95 & 4 & 150,99 & $25 \%$ \\
QUEIJO COALHO & 580,58 & 5 & 116,12 & $19 \%$ \\
LEITE LONGA VIDA- & & & & \\
SOBREMESA & 477,45 & 12 & 39,79 & $7 \%$ \\
BATATA PARA FRITURA & 407,95 & 6 & 67,99 & $11 \%$ \\
GELATINA ZERO ABACAXI & 340,29 & 5 & 68,06 & $11 \%$ \\
\hline
\end{tabular}


Tabela 3. Alimentos industrializados que mais contribuíram para a quantidade de Na ofertada nos almoços da Unidade “3”, situada na cidade de Osasco-SP, no período de 04 a 30/06/2014.

\begin{tabular}{l|c|c|c|c}
\hline \multicolumn{1}{c|}{ ALIMENTO } & $\begin{array}{c}\text { Na per capita } \\
\text { por mês } \\
(\mathrm{mg})\end{array}$ & $\begin{array}{c}\text { Número } \\
\text { de dias de } \\
\text { oferta }\end{array}$ & $\begin{array}{c}\text { Na per } \\
\text { capita por } \\
\text { dia(mg) }\end{array}$ & $\begin{array}{c}\text { Oferta de Na } \\
\text { comparada à } \\
\text { sugestão da } \\
\text { OMS (\%) }\end{array}$ \\
\hline CARNE SECA & 9.019 & 3 & 3.006 & $501 \%$ \\
SHOYU & 1.802 & 18 & 100 & $17 \%$ \\
LINGUIÇA TOSCANA & 1.759 & 1 & 1.759 & $293 \%$ \\
PÃES & 1.540 & 16 & 96 & $16 \%$ \\
LINGUIÇA DE FRANGO & 1.357 & 1 & 1.357 & $226 \%$ \\
SALSICHA HOT DOG & 1.323 & 1 & 1.323 & $220 \%$ \\
AMACIANTE DE CARNE & 1.249 & 17 & 73 & $12 \%$ \\
QUEIJO MUÇARELA & 1.068 & 17 & 63 & $10 \%$ \\
KIBE BOVINO & 886 & 1 & 886 & $148 \%$ \\
\hline
\end{tabular}

Tabela 4. Alimentos industrializados que mais contribuíram para a quantidade de Na ofertada nos almoços da Unidade "4", situada na cidade de São Bernardo do Campo-SP, no período de $13 / 06 / 2014$ a $14 / 07 / 2014$.

\begin{tabular}{|c|c|c|c|c|}
\hline ALIMENTO & $\begin{array}{c}\text { Na per } \\
\text { capita por } \\
\text { mês (mg) }\end{array}$ & $\begin{array}{l}\text { Número } \\
\text { de dias } \\
\text { de oferta }\end{array}$ & $\begin{array}{c}\text { Na per } \\
\text { capita por } \\
\text { dia (mg) }\end{array}$ & $\begin{array}{l}\text { Oferta de } \mathrm{Na} \\
\text { comparada à } \\
\text { sugestão da } \\
\text { OMS }(\%)\end{array}$ \\
\hline TEMPERO GOURMET - CARNES & 7.078 & 9 & 786 & $131 \%$ \\
\hline TEMPERO GOURMET - AVES & 4.611 & 8 & 576 & $96 \%$ \\
\hline LINGUIÇA TOSCANA & 1.467 & 1 & 1.467 & $244 \%$ \\
\hline AMACIANTE DE CARNE & 1.373 & 2 & 686 & $114 \%$ \\
\hline LINGUIÇA DE FRANGO & 1.371 & 2 & 685 & $114 \%$ \\
\hline MOLHO TOMATE REFOGADO & 1.243 & 11 & 113 & $19 \%$ \\
\hline $\begin{array}{l}\text { ESPETO DE FILE DE COXA- } \\
\text { (unidade } \mathrm{c} / 60 \mathrm{~g} \text { ) }\end{array}$ & 1.082 & 1 & 1.082 & $180 \%$ \\
\hline
\end{tabular}




\begin{tabular}{l|c|c|c|c}
\hline \multicolumn{1}{c|}{ ALIMENTO } & $\begin{array}{c}\text { Na per } \\
\text { capita por } \\
\text { mês (mg) }\end{array}$ & $\begin{array}{c}\text { Número } \\
\text { de dias } \\
\text { de oferta }\end{array}$ & $\begin{array}{c}\text { Na per } \\
\text { capita por } \\
\text { dia (mg) }\end{array}$ & $\begin{array}{c}\text { Oferta de Na } \\
\text { comparada à } \\
\text { sugestão da } \\
\text { OMS (\%) }\end{array}$ \\
\hline PASTEL PRONTO PIZZA & 1.046 & 2 & 523 & $87 \%$ \\
HAMBURGUER & 927 & 3 & 309 & $52 \%$ \\
GELATINA MORANGO & 872 & 10 & 87 & $15 \%$ \\
SALSICHA & 847 & 1 & 847 & $141 \%$ \\
KIBE & 778 & 1 & 778 & $130 \%$ \\
KIT FEIJOADA COMPLETA & 742 & 2 & 371 & $62 \%$ \\
MOLHO SOJA & 676 & 6 & 113 & $19 \%$ \\
PÓ P/ MOLHO ESCURO & 578 & 4 & 144 & $24 \%$ \\
\hline
\end{tabular}

Molhos, temperos e carnes processadas aparecem em todas as tabelas deste trabalho como alguns dos principais constituintes do alto teor de sódio em produtos industrializados. Webster, Dunford \& Neal ${ }^{19}$ identificaram esses mesmos elementos em seu estudo realizado na Austrália.

Os itens apontados nas tabelas 1 a 4 também estão de acordo com o encontrado por Ni Mhurchu et al. ${ }^{20} \mathrm{Em}$ seu trabalho, os autores analisaram 44.000 alimentos comprados por 21 mil domicílios do Reino Unido e verificaram que entre os maiores contribuintes de sódio estão o sal de mesa (23\%), a carne processada (18\%), o pão e produtos de panificação (13\%), os produtos lácteos (12\%) e os molhos e patês (11\%). Também identificaram que mais de um terço do sódio adquirido (37\%) vinha de cinco categorias de alimentos: bacon, pão, leite, queijo e molhos.

Nas UANs analisadas no estudo, também apareceu o uso de temperos prontos, amaciante de carnes, gelatina, margarina, azeitona, batata para fritura e nhoque pré-cozido como grandes fornecedores de sódio, pelo uso de alimentos industrializados, conforme se identifica nas tabelas 1 a 4 .

Conhecer esses produtos é importante porque, conforme conclusão de Ni Mhurchu et al. ${ }^{20}$

A segmentação de redução de sódio em um pequeno número de categorias de alimentos e com foco em produtos vendidos nos mais altos volumes pode levar a grandes reduções do sódio disponível para consumo e, portanto, em ganhos para a saúde pública. 
O INTERMAP (Internacional Collaborative Study of Macronutrientes, Micronutrientes and Blood Pressure - estudo internacional realizado entre 1996 e $1999^{21}$ - revelou que a fonte de consumo de sódio varia de acordo com o país estudado, mostrando mais uma vez a importância de se conhecer a origem do consumo para combatê-lo. Tal projeto identificou, por exemplo, que na República Popular da China, a maior parte do consumo de sódio vem da adição do sal na comida caseira (76\%). No Japão, a maior fonte é o uso de molho de soja (63\%) e no Reino Unido (95\%) e nos Estados Unidos (71\% - subestimados por razões metodológicas), a maior contribuição é dos alimentos processados. ${ }^{22}$

É possível que uma alimentação nutritiva, segura, agradável e acessível esteja disponível no prato de todo brasileiro. Mas essa é uma medida que prescinde da união de forças e conhecimento de diversos profissionais, como é o caso de nutricionistas, de profissionais da área de ciências de alimentos, da própria indústria de alimentos e de outras áreas, com ou sem uma ação governamental. Exemplo positivo desse esforço conjunto é o notável programa desenvolvido no Reino Unido para a redução do consumo de sal por parte da população e que tem o apoio do CASH (Consensus Action on Salt and Health), desde 1996, e que trata de convencer os fabricantes de alimentos a reduzir gradualmente o teor de sal de alimentos processados, educar o público a respeito dos danos do sal sobre a saúde humana e evidenciar o impacto de todo esse trabalho na saúde pública. ${ }^{23}$

O sucesso do CASH resultou na formação, em 2005, da WASH (World Action on Salt छُ Health), um grupo apoiado por 527 membros de 95 países e que visa melhorar a saúde da população mundial através da redução do consumo de sal, inclusive aquele incidente sobre produtos industrializados. ${ }^{24}$

Neste trabalho se observou, em diversas ocasiões, que itens iguais foram oferecidos em diferentes unidades, porém de fornecedores diversos, o que implicou oferta distinta de sódio aos comensais. Um bom exemplo dessa situação é a gelatina: 34,64mg/g, 12,71mg/g, 9,60mg/g e 4,78mg/g são algumas medidas dos teores de sódio contidos nas diversas marcas do produto utilizado nas diferentes UANs. Neste caso, o interesse na redução do consumo desse mineral fará com que a leitura do rótulo, por parte do profissional de nutrição responsável pelas compras nos restaurantes, o planejamento de cardápio e a determinação das técnicas de preparo se tornem imprescindíveis para que se ofereçam preparações com menor teor de sódio, sem necessariamente alterar o custo do cardápio.

O trabalho de Dötsch et al. ${ }^{25}$ mostra que $75 \%$ da ingestão de sódio vêm de produtos industrializados e, por isso, autoridades mundiais, como a OMS, têm incentivado a indústria de alimentos a reduzir o nível de sal em seu produto. Diversos projetos identificam que o envolvimento governamental é a chave fundamental para que a redução de oferta de sódio aconteça, quer seja tratando de questões mais gerais, como das mais específicas. A ação que envolve a educação dos indivíduos com relação ao conhecimento dessa informação pode ser considerada como de caráter geral. 
Como de caráter específico, devem ser consideradas as instruções para modificações que devem ocorrer na elaboração dos alimentos industrializados. Isso porque essas medidas dependem da aprovação de leis, com metas globais e transparentes. He, Campbell e Mac Gregor ${ }^{26}$ listam, em seu trabalho, os governos engajados em projetos desse tipo. Estão entre os países citados o Reino Unido, Canadá, Argentina, Brasil, Chile, Costa Rica, Cuba, Guatemala, México, Suriname, Uruguai, África do Sul e a cidade de Nova York.

Patel et al. ${ }^{27}$ estudaram o grau de confiança do consumidor em ações governamentais para a redução de sódio em alimentos processados e concluíram que a maioria dos consumidores (55,9\%) acreditam que seja uma boa ideia a intervenção do governo. É mais um elemento a se considerar, para evitar que ocorram exorbitâncias como as demonstradas nas tabelas 2 a 5 , quando se compara o teor de sódio oferecido aos clientes das UANs com o sugerido pela OMS. A oferta desse mineral chega a ser maior do que o total sugerido, como identificado nos exemplos abaixo:

A unidade 2, por usar amaciante de carne em algumas preparações, ultrapassou mais de $50 \%$ dos 700mg preconizados, já que o consumo de sódio per capita nessa situação foi igual a $1.078 \mathrm{mg}$.

- Na unidade 3, a oferta de quibe bovino foi responsável por 886mg de sódio na porção per capita, o que resultou em $127 \%$ do valor indicado pela OMS.

- Na unidade 4, a oferta de $1.082,16 \mathrm{mg}$ desse mineral se deu por conta do preparo que usou espeto de filé de coxa industrializado, o que representou valor $55 \%$ superior aos $700 \mathrm{mg}$.

- Na unidade 1, a utilização de linguiça de frango promoveu a oferta de 369,09 mg de Na per capita. Somente a quantidade de sódio presente neste alimento representa $53 \%$ da recomendação de consumo diário feita pela OMS.

O conhecimento e estímulo dos administradores de UANs para preparar refeições com menos sódio são armas essenciais para a redução do consumo desse nutriente, o que evita a situação descrita acima. Comprovando essa teoria, o estudo promovido por Ma et al. ${ }^{28}$ relata que a maioria de proprietários e chefs de restaurantes chineses na Filadélfia estariam dispostos e capazes a reduzir o teor de sódio nas refeições, se a demanda de clientes pudesse ser mantida e se a eles fosse fornecido treinamento para a preparação e compra dos alimentos.

Não deixar seus clientes descontentes é uma questão apropriada quando se pensa em alterar o teor de sódio da alimentação oferecida. Porém, havendo interesse, a ciência hoje é capaz de estudar, identificar e até mesmo criar substitutos para o sal. Kremer, Mojet \& Shimojo ${ }^{29}$ estudaram a redução do sal em alimentos do tipo sopa, salada e carne de porco frita, usando molho de soja em substituição, e concluíram que é possível trocar o $\mathrm{NaCl}$ dos alimentos por esse molho, sem perder a intensidade dos sabores e, portanto, a aceitação por parte dos clientes. Dötsch et al. ${ }^{25}$ citam, em seu trabalho, que a investigação sobre a fisiologia dos receptores de paladar do sal é uma área emergente e, com seu desenvolvimento, o alcance na redução de sódio poderá ser ainda maior. 


\section{Conclusão}

Nas UANs, a oferta de sódio oriunda dos alimentos industrializados é alta, o que contribui para uma oferta de preparações com maior teor de sódio aos trabalhadores que fazem as refeições nesses espaços.

Considerando que todas as unidades avaliadas apresentaram resultados semelhantes, faz-se necessário rever as técnicas de preparo utilizadas na UAN com revisão dos ingredientes usados nas preparações. Sugere-se que priorizem o uso de alimentos in natura nas preparações, em detrimento dos produtos industrializados, e que no ato da compra sejam escolhidos aqueles insumos com menor teor de sódio em sua composição.

\section{Referências}

1. Brasil. Ministério da Saúde. Guia alimentar para a população brasileira: promovendo a alimentação saudável. Brasília: Ministério da Saúde; 2005.

2. NORSAL. [acesso em: 21 set. 2013]. Disponível em: http://www.norsal.com.br/main.php?g_ct=sobre

3. Polonia J, Maldonado J, Ramos R, Bertoquin S, Duro M, Almeida C, et al. Determinação do consumo de sal numa amostra da população portuguesa adulta pela excreção urinária de sódio. Sua relação com a rigidez arterial. Revista Portuguesa de Cardiologia, Portugal, 2006; 25(9)801-817.

4. Diretrizes Brasileiras de Hipertensão VI. Revista Brasileira de Hipertensão 2010; 17(1):7-10.

5. Jorge RA, Rodrigues CSC. Papel dos nutrientes na prevenção e no tratamento da osteoporose: [revisão]. Revista Brasileira de Nutrição Clínica 2009; 24(1):66-71.

6. Carneiro MRG. Fatores de risco ambientais para o câncer gástrico: a visão do toxicologista. Caderno de Saúde Pública 1997; 13(1):27-38.

7. Abreu AS, Viana IC, Moreno RB, Torres EAFS. Alimentação mundial: uma reflexão sobre história. Saúde e sociedade 2001; 10(2):3-14.

8. World Health Organization. Salt reduction. [acesso em: mar. 2014]. Disponível em http://www.who. int/mediacentre/factsheets/fs393/en/

9. Instituto Brasileiro de Geografia e Estatística. Pesquisa de orçamentos familiares 2008-2009. Rio de Janeiro: IBGE; 2011.

10. Magalhães T. Padrões alimentares e hipertensão. Revista Factores de Risco 2009; 14:50-53.

11. Ferreira-Sae MC, Gallani MC, Nadruz W, Rodrigues RC, Franchini KG, Cabral PC, et al. Reliability and validity of a semi-quantitative FFQ for sodium intake in low-income and low-literacy Brazilian hypertensive subjects. Health Nutrition 2009; 12(11):2168-2173. 
12. World Cancer Research Fund. Stopping cancer before it starts. Reino Unido, 2012. [acesso em: 26 abr. 2013]. Disponível em: http://www.muchloved.com/partners/wcrf.aspx

13. Andrade ECB, Jesus DC. Avaliação dos teores de sódio em batatas chips e salgadinhos extrusados. Higiene Alimentar 2008; 22:85-99.

14. Sichieri R, Coitinho DC, Coutinho JB, Coutinho WF. Recomendações de alimentação e nutrição saudável para a população brasileira. Arq. Bras. Endocrinol. Metab. 2000; 44(3):227-232.

15. Portal da Saúde. SUS. Redução de sódio. [acesso em: 22 set 2012]. Disponível em: http://dab.saude. gov.br/portaldab/ape_promocao_da_saude.php?conteudo=reducao

16. Abreu ES, Spinelli MGN, Pinto AMS. Gestão de unidades de alimentação e nutrição: um modo de fazer. 2 ed. São Paulo: Metha; 2007.

17. Brasil. Agência Nacional de Vigilância Sanitária. Guia de boas práticas nutricionais: restaurantes coletivos. Brasília: Anvisa; 2014. [acesso em: 29 out. 2015]. Disponível em: http://www.mds.gov.br/ webarquivos/publicacao/seguranca_alimentar/GuiaRestaurantesColetivos.pdf.

18. Borjes LC, Tasca FJ, Zamprogna PE. Alimentos industrializados fontes de sódio utilizados no preparo de refeições em restaurantes comerciais de Chapecó-SC. DEMETRA: Alimentação, Nutrição \& Saúde 2014; 9(1):83-97.

19. Webster JL, Dunford EK, Neal BC. A systematic survey of the sodium contents of processed foods. The American Journal of Clinical Nutrition 2010; 91(2):413-420.

20. Ni Mhurchu C, Capelin C, Dunford EK, Webster JL, Neal BC, Jebb SA. Sodium content of processed foods in the United Kingdom: analysis of 44.000 foods purchased by 21.000 households. The American Journal of Clinical Nutrition 2011; 93(3):594-600.

21. INTERMAP (INTERnacional Collaborative Study of MAcronutrientes, Micronutrientes and Blood Pressure). London: Imperial College, School of Public Health; 2013. [acesso em: 31 jul; 2014]. Disponível em: http://www1.imperial.ac.uk/publichealth/departments/ebs/projects/cdel/intermap/

22. Anderson CAM, Appel LJ, Okuda N, Brown IJ, Chan Q, Zhao L, et al. Dietary sources of sodium in China, Japan, the United Kingdom, and the United States, women and men aged 40 to 59 years: the INTERMAP study. Journal of the Academy Nutrition and Dietetics 2010; 110(5):736-745.

23. CASH - Consensus Action on Salt \& Health. [acesso em: 30 jul. 2014. Disponível em: http://www. actiononsalt.org.uk/about/index.html

24. WASH - World Action on Salt \& Health. [acesso em: 30 jul. 2014. Disponível em: http://www. worldactiononsalt.com/index.html

25. Dötsch M, Busch J, Batenburg M, Liem G, Tareilus E, Mueller R, et al. Strategies to reduce sodium consumption : a food industry perspective. Critical Reviews in Food Science and Nutrition 2009; 49(10):841-851.

26. He FJ, Campbell NR, MacGregor GA. Reducing salt intake to prevent hypertension and cardiovascular disease. Revista Panamericana de Salud Pública 2012; 32(4):293-300. 
27. Patel SM, Gunn JP, Tong X, Cogswell ME . Consumer sentiment on actions reducing sodium in processed and restaurant foods, Consumer Styles 2010. American Journal of Preventive Medicine 2014; 46(5):516-524.

28. Ma GX, Shive S, Zhang Y, Aquilante J, Tan Y, Zhao M, et al. Knowledge, perceptions and behaviors related to salt use among Philadelphia chinese take-out restaurant owners and chefs. Health Promotion Practice 2014; 15(5):638-45.

29. Kremer S, Mojet J, Shimojo R. Salt reduction in foods using naturally brewed soy sauce. Journal of Food Science 2009; 74(6):S255-S262.

Recebido: 16/6/2015

Revisado: 28/10/2015

Aceito: 24/3/2016 